\title{
Pour une classification des témoins de "La Prison d'amour" de François Dassy: paratexte et macrostructure
}

Irene Finotti

\section{(2) OpenEdition}

Journals

Édition électronique

URL : https://journals.openedition.org/studifrancesi/4541

DOI : 10.4000/studifrancesi.4541

ISSN : 2421-5856

Éditeur

Rosenberg \& Sellier

Édition imprimée

Date de publication : 1 avril 2012

Pagination : 69-78

ISSN : 0039-2944

\section{Référence électronique}

Irene Finotti, «Pour une classification des témoins de "La Prison d'amour" de François Dassy: paratexte et macrostructure », Studi Francesi [En ligne], 166 (I | LVI) | 2012, mis en ligne le 30 novembre 2015, consulté le 19 novembre 2021. URL : http://journals.openedition.org/studifrancesi/4541 ; DOI : https://doi.org/10.4000/studifrancesi.4541

\section{(c)}

Studi Francesi è distribuita con Licenza Creative Commons Attribuzione - Non commerciale - Non opere derivate 4.0 Internazionale. 


\section{Pour une classification des témoins de "La Prison d'amour" de François Dassy: paratexte et macrostructure}

Si la Cárcel de amor de Diego de San Pedro ${ }^{1}$ a fait l'objet de plusieurs études, voire de différentes éditions, critiques ou non, depuis le début du siècle dernier ${ }^{2}$, si sa traduction italienne (Carcer d'Amore, Venise, 1514) a été elle aussi éditée en 1986 avec la version catalane (Càrcer d'Amor, Barcelone, 1493)' ${ }^{3}$, une étude complète de la transmission en France de ce roman manque toujours. En effet, les deux traductions françaises - l'une, de la première moitié du XVI ${ }^{\mathrm{e}}$ siècle, par François Dassy, l'autre, de 1552 par Gilles Corrozet - ont diversement attiré l'attention des chercheurs: alors que la transmission de la première est encore objet d'étude ${ }^{4}$, la rédaction de Corrozet a donné lieu à une édition moderne de la part de Véronique Duché-Gavet ${ }^{5}$.

La Prison d'amour de Dassy a pourtant joui d'une grande fortune à la Renaissance, comme le prouve la conservation d'un nombre important de témoins, pas moins de $16^{6}: 11$ manuscrits et 5 éditions.

(1) Ce roman sentimental, publié pour la première fois en 1492 à Séville par Juan Pegnitzer de Nuremberg, Magnus Herbst, Thomas Glockner et Jacobo Cromberger (Madrid, Biblioteca Nacional, I-2134), insère l'histoire des amours de Leriano et Laureola dans un cadre allégorique: le narrateur se promène dans une vallée de la Sierra Morena lorsqu'il rencontre un chevalier - Desir - qui accompagne un homme enchaîné; arrivés à la «Prison d'amour», lieu qui symbolise tous les tourments du captif Leriano, ce dernier supplie l'aucteur de l'aider auprès de la dame qui provoque sa douleur. Le narrateur suivra les deux amants dès leur premier contact jusqu'au refus de la princesse Laureola de continuer la relation et au suicide de Leriano.

(2) Diego de San Pedro, Cárcel de amor, éd. R. Foulché-Delbosc, Barcelona, Biblioteca Hispanica, 1904; D. DE SAn Pedro, Cárcel de amor, éd. M. MenÉndeZ y Pelayo, Orígenes de la novela, vol. II, Madrid, Bailly-Baillière, 1907; DIEGO DE San Pedro, Cárcel de amor, éd. J. Rubió BalaGUER, Barcelona, Ediciones Armiño, 1941; DIEGO DE SAN Pedro, Cárcel de amor, Obras completas, vol. II, éd. K. Whinnom, Madrid, Castalia, 1971; A. Corfis, Diego de San Pedro's "Cárcel de amor". A Critical Edition, London, Tamesis, 1987; DIEGO de San Pedro, Cárcel de amor, éd. C. Parrilla, Barcelona, Crítica, 1995; Diego de San Pedro,
Cárcel de amor. Tractado de amores de Arnalte y Lucenda. Sermón, éd. J. F. Ruiz Casanova, Madrid, Cátedra, 1995.

(3) V. Minervini - M.L. Indini, Càrcer d'amor. Carcer d'amore. Due traduzioni della 'novela' di Diego de San Pedro, Fasano, Schena, 1986.

(4) Cf. M. Dickman Orth, "The Prison of Love": A Medieval Romance in the French Renaissance and its Illustration (B.N. MS fr. 2150), «Journal of the Warburg Courtauld Institutes», 46, 1983, pp. 211-221; I. FINOTTI, Traduire d'une langue à l'autre, traduire de prose en rime: de la "Cárcel de amor" à la "Prison d'amour", "Euvres et Critiques», pp. 112-114.

(5) D. DE SAn Pedro, La Prison d'amour (1552), éd. V. Duché-Gavet, Paris, Champion, 2007; c.r.: M. Colombo Timelli, «Studi Francesi», 153, 2007, pp. 637-638; Mounier, «Réforme, Humanisme, Renaissance», 66, 2008, pp. 141-143; M. N. CANAL, «Vox Romanica», 67, 2008, pp. 337-338.

(6) La recensio des manuscrits de La Prison a permis de découvrir de nouveaux témoins encore récemment: en 1983 Orth ("The Prison of Love...", art. cit.) examinait 7 exemplaires, en 2007 Duché-Gavet (Diego de SAn Pedro, La Prison d'amour (1552), éd. cit.) en énumère 9. Personnellement, j'ai pu ajouter deux autres manuscrits ( $A$ et G2). Il est évidemment impossible d'exclure d'ultérieures découvertes. 
P1 Paris, BnF, nouv. acq. fr. 7552

(ms. autographe)

P2 Paris, BnF, fr. 24382

P3 Paris, BnF, fr. 2150

Cb Chantilly, Musée Condé, ms. 679

O Oxford, Bodleian Library, ms. Rawl. D. 591

G1 Genève, Fondation Martin Bodmer, ms. 149

G2 Genève, BGE - Bibliothèque de Genève, ms. fr. 186

N1 New York, Hispanic Society of America, 706

N2 New York, Hispanic Society of America, 705

A Paris, Arsenal, ms. 3352

B Berkeley, University of California, BANC MS UCB 178

DuP1 Paris, Arsenal, 8 B.L. 29578

DuP2 Paris, BnF, Rothschild, IV.5.7;

éd. 1525, Paris, Antoine Couteau pour Galliot Du Pré éd. 1526, Paris, Antoine Couteau pour Galliot Du Pré

Wolfenbüttel, Herzog August Bibliothek, Lm 875a

Paris, BnF, RES Y2 2350;

Londres, BL, 33.f.1

Bon Chantilly, Musée Condé, III-F-064;

Vienne, ONB, 39.K.37

Arn Paris, BnF, RES Y ${ }^{2} 866$,

Grenoble, BM, F.7292

Ser Chantilly, Musée Condé, III-C-037 éd. 1527, Paris, [Antoine

Bonnemere] éd. 1528, Lyon, Olivier Arnoullet éd. 1533, Paris, [Pierre Sergent]

\section{Datation des témoins}

S'il est toujours vrai que recentiores non deteriores, une datation tout au moins approximative de ces témoins pourrait sinon permettre de reconnaître définitivement le 'meilleur', au moins servir à l'établissement d'un stemma. Aucun manuscrit n'étant daté, des hypothèses peuvent néanmoins être avancées.

P1, vraisemblablement autographe de François Dassy, ne peut pas avoir été copié avant 1522; c'est en effet à cette date que Jacquette de Lansac, dédicataire du manuscrit ${ }^{7}$ et peinte en veuve dans la miniature initiale, perd son mari, Alexandre de Saint-Gelais, chamberlain de Louis XII.

P2 présente divers éléments extratextuels qui peuvent contribuer à sa datation. Dans la panse du $R$ initial enluminé de la Préface (f. $3 \mathrm{r}$ ) se trouvent les armes, d'or au sautoir d'azur, d'Hugues de Loges ${ }^{8}$, seigneur de La Boulaye et de Chailly-sur-Armançon, qui fut lieutenant du gouverneur de Bourgogne et bailli d'Autun sous François $\mathrm{I}^{\mathrm{er}}$, «l'un des cent Gentils-hommes ordinaires de l'Hôtel du Roi» ${ }^{10}$; un cimier à deux oreilles d'âne surmontant le blason et la devise $A$ che coup confirment cette attribu-

(7) P1 porte même les armes de Jacquette, qui est aussi la dédicataire de la traduction de François Dassy (cf. infra).

(8) Il m'est agréable d'exprimer ici mes remerciements et toute ma reconnaissance à M. François Avril à qui je dois l'attribution de ce manuscrit.

(9) G. Thaumas de La Thaumassière, Histoire de Berry, Bourges, 1689, p. 486.

(10) ACHE:COUP à l'intérieur des contours de la haste de la lettre $R$ et encore dans la haste des lettrines initiales miniaturées $P$ [f. 28v], $L$ [f. 39r] et $P$ [f. 47v]; ACE. COVP au f. 23 r. Cf. Inventaire général des monuments et richesses artistiques de la France. Les vitraux de Bourgogne, Franche-Comté et Rhône-Alpes, Paris, Centre national de la recherche scientifique, 1986, p. 30, fig. 15, et H. ABORD, Histoire de la réforme et de la Ligue dans la ville d'Autun, Paris-Autun, Dumoulin, 1855, t. I, p. 175, n. 2. 
tion $^{11}$. Le style des scènes figurant dans le cadre décoré de la même page permet de localiser le manuscrit à Tournai, dont Hugues de Loges fut gouverneur de 1518 à décembre $1521^{12}$. Cette origine expliquerait aussi la forme picarde de la devise. Les armes, au bas de l'encadrement, d'argent à six mains dextres de gueules posées 3, 2 et 1, appartiennent à la seconde femme d'Hugues de Loges, Charlotte Du Mesnil-Simon, qu'il épousa vers $1520^{13}$. Par conséquent, P2 a été fabriqué en 1521 au plus tard. D'après l'analyse détaillée des miniatures de P3 menée par Myra Orth, qui en réfute l'attribution à Godefroy le Batave ${ }^{14}$, ce manuscrit aurait été copié pour Marguerite d'Angoulême (en tout cas, sans aucun doute, pour un membre de la famille de François $\left.{ }^{\text {er }}\right)$ vers $1527^{15}$.

Les armoiries - au verso du premier feuillet - de Françoise d'Alençon circonscrivent la conception de G1 à la période, assez ample, où cette dame a été mariée avec Charles de Bourbon: 1513-1537. Le manuscrit, portant les armes des Bourbon et des d'Alençon ${ }^{16}$ n'est probablement pas dédié au couple mais à la seule femme, comme l'atteste l'épître-dédicatoire. Toujours selon M. Orth, on pourrait réduire cet intervalle aux décennies 1520-1530 sur la base des enluminures. Les miniatures permettraient aussi de faire remonter $O$ aux années $1520^{17}$

Pour ce qui concerne N1, on peut supposer la fourchette 1518-1524. La première date correspond à la naissance du dauphin de France, François, commentée dans les textes qui suivent La Prison d'amour dans ce manuscrit (Genese tres beureux et naissance tres felice de monseigneur le Dauphin F., pp. 277-279, Jugement et pronostication du Roy et de la Reyne pour la nativité dessus figuree, pp. 279-281, De la forme figure et complexion du corps de monseigneur le daulphin, pp. 281-283, Comment il viura en la susdicte disposition de ses malladies et du temps divers, pp. 283-285); quant au terme ante quem, il est vraisemblable que la reine à laquelle le manuscrit est offert dans une dédicace chiffrée ${ }^{18}$ soit Claude de Valois, mère du dauphin François et décédée en 1524.

Selon les inscriptions, dans des graphies du XVI ${ }^{e}$ siècle, au début et à la fin du manuscrit (ff. $1 \mathrm{v}$ et 92v), N2 a appartenu à «Bon Infants Christophe [...] dis» et à «Gilbert Jeny Delinbant de Nemur», que je n'ai pas encore identifiés.

(11) $\mathrm{Au}$ moment de la reddition de la ville à Charles Quint, Hugues de Loges, obligé de quitter Tournai, est comblé de cadeaux par les habitants de la ville: la tapisserie, encore conservée au château de La Boulaye, portant les armes de Loges et datant de 1521, en serait une preuve (cf. H. ABORD, Histoire de la réforme, op. cit., p. 175, n. 2). C'est toujours François Avril qui localise le manuscrit, en trouvant une confirmation dans sa ressemblance avec deux autres manuscrits tournaisiens: les Heures Trameries à l'usage de Tournai (Les Enluminures, cat. 9, 2000, $\left.n^{\circ} 25\right)$ et la Chronique des évêques de Tournai (Tournai, Bibliothèque du chapitre cathédral, ms. B. 1, reprod. dans D. VANWIJNSBERGHE, De fin or et d'azur. Les commanditaires de livres et le métier de l'enluminure à Tournai à la fin du Moyen Âge (XIV"$X V^{e}$ siècles), Louvain, 2001, fig. 127).

(12) «Charlotte Dumesnil-Simon femme de messire Hugues de Loges, chevalier seigneur de Chailly et de La Boulaye, [...] lequel, à cause de sa femme fit partage avec François Dumesnil-Simon son beau-frère \& eut en son lot le tiers de la châtellenie de Beaujeu \& seigneurie des Quartiers Rogers, le 7 février $1520 »$ (G. Thaumas DE LA Thaumassière, Histoire de Berry, op. cit., p. 486).
(13) P. Durrieu, «Dominus Illuminatio mea». Les vingt 'rondeaux' dessinés de Godefroy le Batave dédié au roi François I I, Paris, 1922, pp. 22-23; M. Dickman OrTh, Progressive Tendencies in French Manuscript Illumination, 1515-30. Godefroy le Batave and the 1520s Hours Workshop, Ph.D. dissertation, New York University, Institute of Fine Arts, 1976.

(14) ORTh, "The Prison of Love".., art. cit., pp. 217-221.

(15) Un blason parti au premier d'azur aux trois fleurs de lys d'or à la bande de gueules, au second d'azur aux trois fleurs de lys d'or à la bordure de gueules chargée de huit besants d'argent.

(16) ORTH, "The Prison of Love"..., art. cit., n. 13 et p. 213. fra).

(17) Les initiales de la reine sont $C . d$. $u$. (cf. in -

(18) N'ayant pas pu voir jusqu'à aujourd'hui ce manuscrit, je m'appuie sur les informations contenues dans Ch. B. FAulHaber, Medieval Manuscripts in the Library of the Hispanic Society of America. Religious, Legal, Scientific, Historical, and Literary Manuscripts, New York, The Hispanic Society of America, 1983, n. 705. 
Restent à dater 4 manuscrits $(C h, G 2, A, B)$, pour lesquels aucun élément extratextuel probant n'a été mis en lumière jusqu'à présent.

Par ailleurs, les dates des cinq éditions - 1525, 1526, 1527, 1528, 1533 - montrent que la tradition imprimée est contemporaine de la manuscrite.

\section{Notes pour un stemma}

La complexité de la transmission de La Prison d'amour est accrue par la stratification de plusieurs mains qui n'ont pas hésité à intervenir sur le texte, souvent de façon marquante. Dans la phase actuelle de mes recherches, je ne peux pas exclure que certains témoins constituent des codices descripti; d'ailleurs une collation encore approximative laisse supposer que les quatre dernières éditions (DuP2, Bon, Arn, Ser) pourraient être de simples copies l'une de l'autre. Ce qui est évident, c'est que des variantes, parfois significatives, au niveau du paratexte et de la macrostructure, touchent plusieurs manuscrits, ainsi que les deux premières éditions.

Je me servirai encore d'éléments extratextuels pour essayer de reconstruire certaines relations entre les témoins ou pour déterminer des lieux critiques. À ce propos, un distinguo préliminaire s'impose: alors que l'analyse de la structure du roman peut être appliquée à tous les témoins, le paratexte, à cause des caractères propres aux imprimés, ne nous permettra pas toujours de mettre en relation les manuscrits avec les éditions.

\begin{tabular}{|c|c|c|c|c|c|c|c|c|c|c|c|c|c|c|c|c|c|}
\hline & & & & & & & & & & & & & & & & \\
\hline & & P1 & $P 2$ & P3 & $\mathrm{Cb}$ & $O$ & G1 & $G 2$ & N1 & N2 & $A$ & $B$ & DuP1 & DuP2 & Bon & Arn & Ser \\
\hline \multirow{2}{*}{ INTITULÉ } & $\begin{array}{l}\text { La Prison } \\
\text { d'amour }\end{array}$ & & & $\mathrm{X}$ & & & & & & & & & $\mathrm{x}$ & $\mathrm{x}$ & $\mathrm{X}$ & $\mathrm{X}$ & $\mathrm{X}$ \\
\hline & $\begin{array}{l}\text { rappel } \\
\text { du contenu }\end{array}$ & & & & & $\mathrm{x}$ & & & & & & & $\mathrm{x}$ & $\mathrm{x}$ & $\mathrm{x}$ & $\mathrm{X}$ & $\mathrm{x}$ \\
\hline \multicolumn{2}{|c|}{ RUBRIQUE de la préface } & & & & & & $\mathrm{x}$ & & $\mathrm{X}$ & & $\mathrm{X}$ & $\mathrm{X}$ & $\mathrm{X}$ & $\mathrm{X}$ & $\mathrm{X}$ & $\mathrm{X}$ & $\mathrm{X}$ \\
\hline \multicolumn{2}{|c|}{$\begin{array}{l}\text { PRÉFACE } \\
\text { (= épître dédicatoire) }\end{array}$} & $\mathrm{X}$ & $\mathrm{X}$ & & & & $\mathrm{X}$ & & $\mathrm{X}$ & & $\mathrm{X}$ & & $\mathrm{X}$ & $\mathrm{x}$ & $\mathrm{X}$ & $\mathrm{X}$ & $\mathrm{X}$ \\
\hline
\end{tabular}

Comme on peut le remarquer dans ce tableau, toutes les éditions portent sur la première page, selon les habitudes de l'époque, un intitulér ${ }^{19}$ qui associe à ce qui à nos yeux constitue le véritable titre (La Prison damour) une bref rappel du contenu: laquelle traicte de lamour de Lerian et | Laureolle/faict en espaignol/ puis I translate en tusquan et naguerre I en language francois Ensemlble plusieurs choses sin// I gulieres a la louenge I des dames (DuP1). Par contre, lorsque dans les manuscrits on trouve le titre du roman, c'est seulement parce qu'un possesseur l'a ajouté dans un espace resté blanc $^{20}$. $O$ et $P 3$ sont les seuls manuscrits qui ont gardé un titre tracé par le copiste ou le rubricateur ${ }^{21}$ : mais, alors que dans $O$ on fait allusion aux deux protagonistes de l'histoire (Sensuyt lee [sic] liure de Leriano Et la dame Laureolle. Et la mort <...> [grat-

(19) Je ne m'arrête pas sur les éléments purement bibliographiques (adresse du libraire, colophon, privilège, table des matières) qui seront examinés à part.

(20) C'est le cas de P2, G1, N2, A et B.
(21) Dans ces deux manuscrits le titre semble avoir été copié avec la même encre (rouge dans $O$, bleue dans $P 3$ ) et par la même main que les titres de chapitre. 
tage] Leriano), le titre de P3, Carcer Damour, fait supposer un lien avec la tradition italienne ou espagnole ${ }^{22}$.

Les mêmes informations sur l'origine de la traduction française, ainsi que sur le contenu, qu'on lit dans les titres des éditions, sont exprimées à nouveau dans tous les imprimés, mais aussi dans quatre manuscrits $(G 1, N 1, A, B)$, dans une rubrique précédant la Préface ou le début du texte quand celle-ci manque: Ce present liure a este translate de | langaige tosquan florentin en francois | Et traicte de lamour de leriano A | laureolle fille du Roy de macedoyne $(A)$. Quoique nous ne puissions pas exclure que d'autres manuscrits aient possédé cette rubrique - éventuellement perdue à cause de sa position liminaire -, on peut supposer une parenté entre les témoins qui l'affichent.

La présence/absence de la Préface donne aussi quelques informations sur les rapports entre les témoins. Comme le prouvent l'acrostiche initial dans $P 1$, où apparaît le nom de Jacquette de Lansac, ainsi que ses armes et ses devises («En bon lieu» et «Sola in patria») ${ }^{23}$ au bas de la miniature dans l'encadrement du f. $1 \mathrm{v}^{24}$, la Préface est dédiée à cette dame. Les clichés de l'épître dédicatoire sont respectés, la captatio benevolentiae, le topos modestiae, et notamment l'offrande de

ce petit livret jadis converty de langue castillagne et espaignolle en tosquan florentin par ung ferrarois, mon bon et singulier amy, des mains duquel, en ce premier voyage que le trescrestien roy Françoys premier de ce nom, nostre seigneurs, a fait en Lombardie pour la conqueste de son estat ultramontain, ay recouvert $(P 1$, f. 2 r).

Selon des exigences liées aux dédicataires, ce prologue-épître, adressé à une dame dont le nom n'apparaît pas, est éliminé par certains copistes, tandis que d'autres le conservent.

$P 1$, on l'a vu, contient l'épître. Il est suivi par P2, G1, N1, $A$ et par toutes les éditions, alors que dans $P 3, C h, O, G 2, N 2$ et $B$ la Préface manque. Cette suppression correspondrait au dessein du copiste, et non pas à un accident matériel, dans $P 3$, $O$ et $G 2$ qui ne semblent pas avoir subi de mutilations au premier cahier ${ }^{25}$. Dans $B$, si la Préface avait existé, elle aurait été placée probablement après la rubrique $\mathrm{Ce}$ present liure a este translate..., comme dans tous les autres cas où les deux - rubrique et Préface - apparaissent. Quant à N2 et Ch la possibilité qu'un feuillet contenant la Préface soit perdu est à envisager.

$P 1$ propose au $\mathrm{f} .1 \mathrm{v}$ un acrostiche de huit vers: les premières lettres de chaque vers, en rouge, composent le nom de la dame à qui le traducteur voue ses efforts, mais d'autres caractères rouges, à l'intérieur du premier quatrain, révèlent le nom de Francois Dacy ${ }^{26}$.

Illustre dame, franc sejour de noblesse $\mathrm{e}^{27}$,

$\underline{A}$ qui sur toutes je dois honneur sans vice

(22) Quoique carcere soit attesté déjà au $\mathrm{XII}^{\mathrm{c}}$ siècle et encore au XVI ${ }^{\mathrm{e}}$ (FEW II-1, 363b).

(23) Cf. H. A. Omont, Catalogue général des manuscrits français. Nouvelles acquisitions françaises, Paris, 1900, p. 167.

(24) Les enluminures et les bois gravés mériteront une analyse détaillée qui ne peut pas trouver sa place ici.

(25) D'après l'analyse codicologique de Mme Marie-Pierre Laffitte de la BnF, de M. Bruce Barker-Benfield de la Bodleian Library et de Mme Paule Hochuli Dubuis de la BGE de Genève que je remercie vivement. Contrairement à ce que dit
H. Aubert, Notices sur les manuscrits Petau, Paris, 1911, pp. 162-163, les cahiers de G2 sont complets (9 quaternions et un ternion final).

(26) "The Prison of Love"..., art. cit., p. 213, n. 7, voit encore un renvoi au traducteur dans la lettre-dédicace, à la suite des mots cités supra: «Et voyant que d'asséz [Dassy] belle matiere traictoit [suj.: le petit livret], mesmes pour jeunes dames, l'ay entreprins mectre et translater dudit ytalien en nostre vernacule et familiere langue françoise $[\ldots] \gg$, f. $2 \mathrm{r} / \mathrm{v}$.

(27) Je souligne les caractères rouges. 
En bon vouloir d'afection expresse.

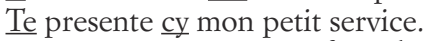

De vray je sçay que c'est faict de novice.

$\underline{\text { L'an }}$ dit pourtant qu'accepter peu de chose

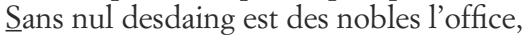

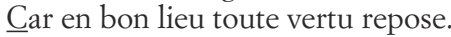

Aucun des autres témoins ne reproduit ce bref poème, qui définit sans hésitation les actants d'un échange - la donation du petit livre -, dont la Préface rend compte plus discrètement.

Néanmoins, en supposant que la devise Celer naper ${ }^{28}$, placée dans un phylactère au centre de l'encadrement du f. $1 \mathrm{v}$ de $P 1$ et reproduite à la fin de la Préface, identifie elle aussi le traducteur, il faut remarquer que $P 2$ est le seul témoin qui la conserve après l'épître dédicatoire.

Pour terminer l'analyse du paratexte, je dois enfin accorder quelques lignes au ms. N1. Comme on le verra, ce n'est pas seulement par son aspect qu'il se démarque des autres: peu soigné, il ne présente aucune décoration sinon quelques lettrines initiales calligraphiées. Dans la reliure actuelle, La Prison d'amour suit un Indice delle Medaglie $e^{29}$ qui se termine à la page 20. La Préface de notre roman, précédée de sa rubrique habituelle, ne débute que vers la moitié de la page 23 ; la page $21^{30}$ est en effet occupée par le petit poème suivant:

Peste n'a lieu ou bon aer est en regne,

Guerre n'a cours ou Dieu veult tenir rene,

Famine n'a ou le bon blé n'est cher:

Sont troys cousteaulx que Dieu faict bien trancher.

Remede:

Foy, charité, esperance en ce lieu

Et ferme amour avecq volunté de Dieu;

Ces troys vertuz nous servent de chasteaulx

Et de fourreaulx pour mectre ses cousteaulx.

La graphie et l'encre semblent bien être les mêmes que dans le reste du texte.

Juste après ces vers, deux lignes attirent encore notre attention: il s'agit d'un bref texte chiffré composé de lettres de l'alphabet latin et de symboles qui rappellent l'alphabet grec:

$<$ ne $>$ manger que une fois par iour aller sans linge $\mid$ et estre solitaire. dées $^{31}$ :

Pareillement, les 7 lignes qui se lisent en haut de la page 23 ont dû être déco-

En reuerence honneur et contemplacion I de la foi fermete et amour que iai a toi mamie I ma femme et ma roine genieure ${ }^{32}$ [?] C.d.u. ${ }^{33}$ que ie I tiens plus chere que moi mesmes iai pour lamour I de toi prins le double [?] et extraict du liure I et traicte des deux amans leriano et laulreole.

(28) À ce jour, on n'a pas encore réussi à l'interpréter (cf. V. DuCHÉ-GAVET, Si du mont Pyrenée / N'eussent passé le haut fait... Les romans sentimentaux traduits de l'espagnol en France au XVI' siècle, Paris, Champion, 2008, p. 83; ORTH, "The Prison of Love"..., art. cit., p. 213, n. 7).

(29) Remontant au XVIII' siècle.
(30) La page 22 est blanche.

(31) Les deux énigmes ont été résolus par Brian Dutton et les solutions publiées par Charles B. Faulhaber (op. cit., n. 706).

(32) Je corrige la transcription (gendeure) de Brian Dutton.

(33) Cf. supra. 
Elles restent toutefois à interpréter: si la dédicataire a été vraisemblablement décryptée, qui est l'auteur de cette énigme? Et pourquoi il l'aurait élaborée?

D'après le ms. autographe, La Prison d'amour compte 47 chapitres. Cette subdivision correspond à celle du texte-source italien, sauf au chap. 9 où François Dassy réunit deux chapitres (L'auctore et L'auctore a Laureola):

Chap. 1 - L'acteur

Chap. 2 - Le prisonnier a l'aucteur

Chap. 3 - Responce de l'aucteur a Leriano

Chap. 4 - L'acteur

Chap. 5 - L'acteur a Laureole

Chap. 6 - Responce de Laureole a l'acteur

Chap. 7 - L'acteur

Chap. 8 - Carte de Leriano a Laureole

Chap. 9 - L'acteur

Chap. 10 - Responce de Laureole a l'acteur

Chap. 11 - L'acteur

Chap. 12 - L'acteur a Leriano

Chap. 13 - Responce de Leriano

Chap. 14- Lettre de Leriano a Laureole

Chap. 15 - L'acteur

Chap. 16- Lettre de Laureole a Leriano

Chap. 17 - L'aucteur

Chap. 18- Lettre de Perseo a Leriano

Chap. 19- Responce de Leriano

Chap. 20 - L'aucteur

Chap. 21 - Leriano au roy

Chap. 22 - L'aucteur

Chap. 23 - L'aucteur a Leriano

Chap. 24 - L'aucteur

Chap. 25 - Lettre de Leriano a Laureole
Chap. 26-L'aucteur

Chap. 27 - Carte de Laureole a Leriano

Chap. 28-L'aucteur

Chap. 29- Le cardinal au roy

Chap. 30 - Responce du roy

Chap. 31 - L'aucteur

Chap. 32 - La royne a Laureole

Chap. 33 - L'aucteur

Chap. 34 - Lettre de Laureole au roy

Chap. 35-L'aucteur

Chap. 36-Leriano a ses chevaliers

Chap. 37 - L'aucteur

Chap. 38 - Lettre de Leriano a Laureole

Chap. 39-L'aucteur

Chap. 40 - Lettre de Laureole a Leriano

Chap. 41 - L'aucteur

Chap. 42 - Leriano a Theseo

Chap. 43 - Vingt raisons de Leriano par lesquelles il veult monstrer les hommes tenuz aux femmes

Chap. 44 - Preuve par exemple de la bonté des femmes

Chap. 45 - L'acteur se torne a l'istoire

Chap. 46 - Plaincte de la mere de Leriano

Chap. 47 - L'aucteur

Aucun des autres témoins de La Prison ne suit fidèlement $P 1$.

C'est $B$ qui s'en éloigne le plus: la réorganisation de la matière est ici la conséquence d'une réduction marquée du texte dans la dernière partie du roman. Du chap. $42, B$ ne conserve que le tout début, où Leriano, au lieu de répondre aux accusations que Theseo avait portées aux dames ${ }^{34}$, l'invite simplement à partir. Les chap. 43-46 - dans lesquels Leriano continue sa célébration du sexe féminin ${ }^{35}$ à la fin de laquelle sa mère le rejoint à son chevet pour exprimer sa douleur - sont entièrement supprimés. Le chap. 47 est disloqué: la première partie se joint au chap. 42 pour former une seule unité, tandis que la fin forme un chapitre à part reprenant le titre de P1 (L'aucteur). Aucun des autres textes conservés ne dérive de $B$.

En collationnant les quatorze autres témoins, j’ai repéré trois types de variantes par rapport à $P 1$. Un chapitre peut être coupé en deux: c'est le cas du 9, du 22 et du 37; deux chapitres peuvent se fondre en un seul: 4+5, 22+23; ailleurs le titre est supprimé mais le découpage reste. Le tableau suivant résume ces variantes ${ }^{36}$.

(34) Dans P1 le chap. 42 est une longue défense des femmes de la part de Leriano, qui va «monstrer par quinze pointz comme grandement errent ceulx qui en se sexe mectent leur langue» $[76 \mathrm{v}-77 \mathrm{r}]$.

(35) «Par vingt raisons $[. .$.$] veult faire aparoir$ que les hommes leur sont moult tenuz et obligéz, en allegant divers exemples de leur bonté» [77r].

(36) Mis en évidence par le gras dans le tableau qui précède. 
1 Introduction Chap. 9

d'une coupure Chap. 22

P3, Ch, N2, DuP1, DuP2, Arn, Bon, Ser

Chap. 37

$\mathrm{Ch}, \mathrm{N} 2$

P3, Ch, N2, DuP1, DuP2, Arn, Bon, Ser

2 Suppression

Chap. $4+5$

N1

de coupure

Chap. $22+23$

G1

3 Suppression du

Chap. 1

titre de chapitre Chap. 5

Chap. 22

P2, G1, G2, N1, A, B, DuP1, DuP2, Arn, Bon, Ser

Chap. 23
Chap. 43

P2, G1, G2, A

P2, G2

P2, O, G2, N1, A

P2, O, G2, N1

Dans les trois cas de figure, les variantes pourraient dépendre d'une intervention autonome de chaque copiste ou éditeur; le découpage s'expliquerait par le changement des destinataires, des modalités ou des sujets de l'énonciation: au chap. 9 L'aucteur relate d'abord les événements (pour le lecteur), et s'adresse ensuite à Laureole; au chap. 22 la narration de L'aucteur est interrompue par une invocation directe à Perseo $^{37}$; le glissement d'un sujet à un autre (la prise des faux témoins / les passions de Leriano) serait à l'origine du dédoublement du chap. 37. À l'inverse, la fusion des chap. 4 et 5 , ainsi que des chap. 22 et 23 paraît légitimée par le fait que c'est toujours L'auteur qui s'exprime.

Que les témoins dont il est question aient éliminé indépendamment les titres de certains chapitres, ne me paraît pas tout à fait improbable non plus. Le titre du chap. 1 peut être considéré superflu, à plus forte raison si l'incipit est précédé par une rubrique, comme dans le cas de G1, N1, $A, B$; l'intitulé du chap. 43 n'est pas indispensable non plus: c'est toujours Leriano qui s'adresse à Theseo ${ }^{38}$. Par contre la suppression dans P2 et G2 du titre du chap. 22 (L'aucteur), indiquant le passage de la parole de Leriano au narrateur, me paraît plus significative.

En raison de ces constatations, je suppose que, si tous les témoins ont pu séparément introduire une ou plusieurs de ces variantes structurelles, la somme de ces modifications dans plus d'un manuscrit révèle des groupements. J'ai donc isolé $C h$ et $N 2$ - où les chapitres sont au nombre de 51 - pour le premier groupe de variantes; P2 et G2 - qui, par rapport à $P 1$, concordent dans la suppression de 5 titres - pour le troisième.

La collation des titres de chapitre donne aussi quelques renseignements, malgré les nombreuses variantes à l'intérieur même des familles que j'ai reconnues jusqu'ici. Seules les 4 dernières éditions (DuP2, Bon, Arn, Ser) forment de façon évidente un groupe à part avec des variantes communes.

Une variante peut aussi faire supposer un lien entre toutes les éditions et $G 1^{39}$ :

Chap. 2, P1: Le prisonnier a l'aucteur > G1, DuP1, DuP2, Arn, Bon, Ser: Le prisonnier declare les choses dessusdit[es].

Une autre leçon commune regroupe l'autographe et $P 2$ en opposition aux autres manuscrits et éditions: l'intitulé du huitième chapitre comprend en effet un hispanisme (Carte [= 'lettre'] de Leriano a Laureole) que seulement P2 conserve ${ }^{40}$.

(37) Perseo est le sujet de la dispute entre le roi et Leriano, rapportée par L'aucteur au chap. 22.

(38) J'ai déjà discuté la question des chap. 5 et 23: un rapport doit exister entre la suppression des titres (en N1 et G1) et la fusion, dans d'autres témoins, de ces chapitres avec ceux qui les précèdent.
(39) Il est pourtant difficile de trouver d'autres variantes significatives communes à ces 6 témoins.

(40) J'avais déjà supposé une possible relation entre $P 1$ et $P 2$ lorsque j'avais remarqué que seuls ces deux manuscrits portent la devise du traducteur après la Préface (cf. supra). 
Enfin, dernière remarque à propos des titres de chapitre, $C b$ se révèle souvent plus précis ou descriptif que les autres témoins. Voilà trois exemples:

Chap. 20, P1: L'acteur $>$ Ch: L'acteur descript le combat par Lerian a Perseus.

Chap. 21, P1: Leriano au roy $>C h$ : Lerian se complainct au roy $<$ de $>$ l'injure a luy faicte de sa victoire contre Perseus.

Chap. 22, P1: L'acteur $>C h$ : Responce du roy a Lerian et Perseus envoya troys faulx tesmoings au roy pour tesmongner son accusation.

La Prison d'amour de François Dassy, comme ses sources directe et indirecte, est au moins partiellement un roman épistolaire. Les dix épitres, correspondant aux chap. 8, 14, 16, 18, 19, 25, 27, 34, 38, 40, alternent avec la narration de L'aucteur. Lettres d'amour (entre Leriano et Laureole), de défi (entre Leriano et Perseo, l'autre prétendant de la princesse) ou de supplication (Laureole au roi), elles se retrouvent dans tous les témoins du roman français et constituent un lieu particulièrement significatif de collation, non pas par leur nombre, qui ne varie jamais ${ }^{41}$, ni par la position qu'elles occupent, mais à cause de l'alternance de forme, en prose dans certains témoins, en vers dans d'autres.

Dans un article qui vient de paraître ${ }^{42}$, j'ai montré que les 16 témoins du roman de Dassy se répartissent en trois groupes selon qu'ils présentent la version avec toutes les lettres en proses $(P 1, P 2, O, G 1, G 2, A, B)$, les épîtres en vers $(P 3, N 2$, Ch et $D u P 1)$ ou encore, et c'est le cas des 4 dernières éditions ( $D u P 2, A r n, B o n, S e r), 7$ lettres en prose et 3 en rime. N1 se distingue encore une fois proposant toutes les épîtres en prose suivies par la rédaction en vers ${ }^{43}$.

On pourrait représenter cette situation de la façon suivante:

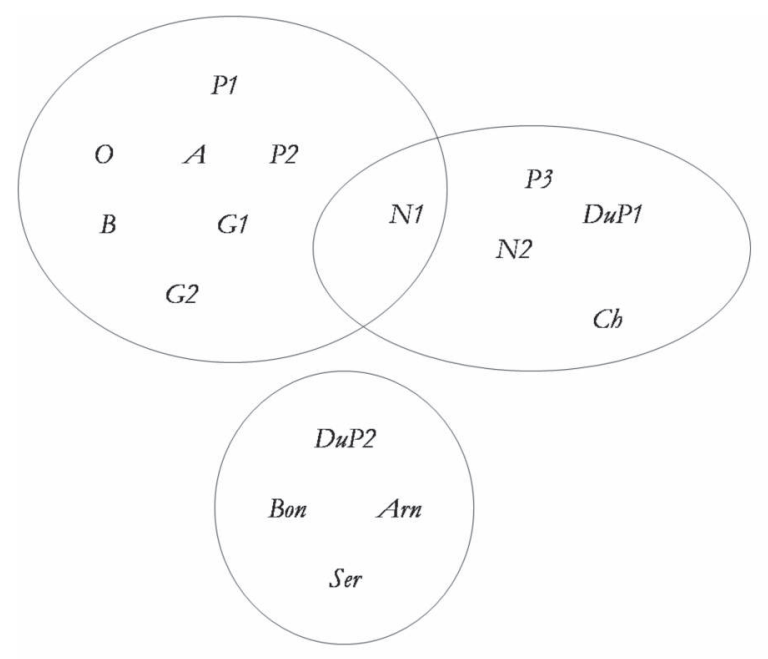

(41) Exception faite pour $A$ qui est inachevé (au recto du f. 39 le chap. 35 s'interrompt).

(42) I. FinotTi, Traduire d'une langue à l'autre...,
(43) Pour plus de détails sur les hypothèses de filiation qui résultent de l'examen des lettres, je renvoie à mon article Traduire d'une langue à l'autre..., art. cit. 


\section{Conclusion}

Même à la lumière de cette première collation qu'on pourrait définir 'superficielle', l'embrouillement de la tradition de La Prison d'amour est encore loin d'être démêlé, les rapports de dépendance entre les témoins ne sont pas définis non plus; mais des alliances, sinon de filiations, ont été esquissées.

Mon but était ici de rendre compte d'une première phase de recherche, sans nullement prétendre épuiser le sujet. À ce jour, les résultats, que j'ai ici réunis dans un tableau récapitulatif, ainsi que les datations des témoins - elles aussi encore susceptibles de précisions - permettront d'entreprendre la collation du texte sur des bases plus sûres.

Familles ou sous-familles

G1, DuP1, DuP2, Bon, Arn, Ser DuP2, Bon, Arn, Ser

N1, N2, P3, Ch, DuP1

$P 1, P 2, A, G 1, G 2, N 1, O, B$

$A, B, G 1, N 1$,

$P 1, P 2$

$\mathrm{Cb}, \mathrm{N} 2$

$P 2, G 2$

$O, B, G 2, P 3, N 2$ ?, $C h$ ?
Variantes communes

«Le prisonnier declare les choses dessusdit[es]» 7 lettres en prose +3 en vers / titres de chapitre Lettres en vers

Lettres en prose

Rubrique précédant la Préface

Devise et «Carte»

51 chapitres

Suppression de 5 titres de chapitre

Suppression de la Préface/dédicace 